\title{
The Impact of Different Plasma Glucose Levels on Heart Rate in Experimental Rats With Acute Myocardial Infarction
}

\author{
Guo-Zhong Pan ${ }^{\text {a, d }}$, Jing Xie ${ }^{\text {a, d }}$, Xiao-Fang Tian ${ }^{\mathrm{b}}$, Shi-Wei Yang ${ }^{\mathrm{c}, ~ e}$, \\ Yu-Jie Zhou ${ }^{\mathrm{c}}$
}

\begin{abstract}
Background: The aim of the study was to evaluate the impact of different plasma glucose levels on heart rate (HR) in experimental rats with acute myocardial infarction (AMI).

Methods: One hundred and twenty-one male Wistar rats were randomly divided into AMI group $(\mathrm{n}=70)$ and sham-operation group (n =51). Both groups had low, normal and high glucose levels, respectively. In the former group, hypertonic glucose was injected into the rats to make their blood glucose levels above $16 \mathrm{mmol} / \mathrm{L}$ and insulin below $3.3 \mathrm{mmol} / \mathrm{L}$; then, the left anterior descending artery was ligated. In the later group, the models of different blood glucose levels were the same as the former ones, but false operations, thread without ligating, were given to the rats. Electrocardiogram and troponin I (TnI) confirmed that the models were prepared successfully. Electrocardiogram expression of AMI was the formation of Q-wave in over three adjacent leads and abnormal elevation of TnI.

Results: The HR of the rats in the hypoglycemic group is higher than that of the hyperglycemic group and normal blood glucose group before AMI $(\mathrm{P}<0.05)$. The HR of the hyperglycemic rats is higher than that of the hypoglycemic group and normal blood glucose group after AMI $(\mathrm{P}<0.05)$. In the hypoglycemic group, the HR of the rats who suffered from AMI was lower than that of the rats of the sham group $(\mathrm{P}<0.05)$.
\end{abstract}

Manuscript accepted for publication August 23, 2016

${ }^{a}$ Dongzhimen Hospital Eastern Affiliated to Beijing University of Chinese Medicine, Beijing, China

${ }^{b}$ Beijing Liangxiang Hospital Affiliated to Capital Medical University, Beijing, China

'Beijing Anzhen Hospital Affiliated to Capital Medical University; Beijing Institute of Heart, Lung and Blood Vessel Disease; The Key Laboratory of Remodeling-Related Cardiovascular Disease, Ministry of Education, Beijing, China

${ }^{\mathrm{d}}$ These authors contribute equally to the work.

${ }^{\text {e}}$ Corresponding Author: Shi-Wei Yang, 12th Ward, Department of Cardiology, Beijing Anzhen Hospital Affiliated to Capital Medical University; Beijing Institute of Heart, Lung and Blood Vessel Disease; The Key Laboratory of Remodeling-related Cardiovascular Disease, Ministry of Education; No. 2 Anzhen Street, Chao Yang District, Beijing 100029, China.

Email: jackydang@163.com

doi: http://dx.doi.org/10.14740/cr488w
Conclusion: Hypoglycemia allows faster HR and the HR in the rats with hyperglycemia is higher than that in the rats with hypoglycemia among the AMI rats.

Keywords: Hyperglycemia; Hypoglycemia; Acute myocardial ischemia; Heart rate

\section{Introduction}

Although hyperglycemia on admission is a powerful predictor of survival in patients with acute coronary syndrome (ACS), intervention to normalize glycemia has yielded inconsistent results. Indeed, recent large randomized controlled trials have failed to show a significant decrease in mortality with intensive glycemic control, or have even shown an increased mortality risk. We have previously reported that in older patients with acute myocardial infarction (AMI), increased as well as mildly decreased admission fasting plasma glucose (FPG) levels could predict higher in-hospital and 3-year mortality $[1,2]$. There was a striking U-shaped relationship between admission FPG levels and all-cause mortality. These results have contributed to confusion regarding optimal glycemic control targets in patients with ACS. However, the pathophysiological mechanisms are unclear. Epidemiological studies have shown that resting heart rate (HR) is a predictor of all-cause mortality and cardiovascular (CV) mortality in subjects with as well as without diagnosed CV disease, and the effect is independent of traditional $\mathrm{CV}$ risk factors [3]. Increased HR is associated with a poor prognosis, and the importance of resting HR as a risk factor in the general population is recognized by the European Guidelines on CV Prevention [4]. In the study, we aimed to evaluate the impact of different plasma glucose levels on HR in experimental rats with AMI.

\section{Materials and Methods}

\section{Animals}

For this experiment, male adult Wistar rats weighing 260 - 320 
Table 1. The Results of Tnl of the Different Groups

\begin{tabular}{lll}
\hline Groups & N & TnI \\
\hline MH & 8 & $77.97 \pm 26.53^{\mathrm{a}}$ \\
MN & 10 & $72.49 \pm 27.64^{\mathrm{a}}$ \\
ML & 13 & $69.93 \pm 27.27^{\mathrm{a}}$ \\
SH & 11 & $2.88 \pm 2.37$ \\
SN & 12 & $2.04 \pm 2.46$ \\
SL & 7 & $2.45 \pm 2.61$ \\
Total & 61 & $38.22 \pm 40.16$ \\
\hline
\end{tabular}

aCompared with the sham groups, the AMI groups had a much higher Tnl, which was statically significant $(P<0.05)$. $\mathrm{MH}$ : myocardial infarction high glucose level; MN: myocardial infarction normal glucose level; ML: myocardial infarction low glucose level; SH: sham-operated high glucose level; SN: sham-operated normal glucose level; SL: shamoperated low glucose level.

$\mathrm{g}$ were used in accordance with recommended guidelines on the care and use of laboratory animals issued by the Chinese Council on Animal Research. The study was approved by the ethics committee of the Key Laboratory of Chinese Internal Medicine (Beijing University of Chinese Medicine), Ministry of Education.

\section{Establishment of experimental rat models of different blood glucose levels}

The healthy adult male Wistar rats $(n=121)$, receiving food and water ad libitum in 1 week of acclimation, were divided randomly into AMI group $(\mathrm{n}=70)$ and sham-operation group $(\mathrm{n}=51)$. Both the two groups had low $\left(\mathrm{n}_{\text {AMI group }}=19, \mathrm{n}_{\text {Sham- }}\right.$ operation group $=19)$, normal $\left(\mathrm{n}_{\mathrm{AMI} \text { group }}=23, \mathrm{n}_{\text {Sham-operation group }}=\right.$ $13)$ and high $\left(n_{\text {AMI group }}=28, n_{\text {Sham-operation group }}=19\right)$ glucose levels, respectively. The rats in the high glucose level group were injected hypertonic glucose to make their blood glucose levels above $16 \mathrm{mmol} / \mathrm{L}[5,6]$. Likewise, the rats in the low glucose level group were injected insulin to make their blood glucose levels below $3.3 \mathrm{mmol} / \mathrm{L}$.

\section{Establishment of experimental rat models of AMI with different blood glucose levels}

When the rats were in the ideal blood sugar levels, the rats were anesthetized by intraperitoneal injection of $1 \%$ sodium pentobarbital at $50 \mathrm{mg} / \mathrm{kg}$, then the back was fixed, the left chest was prepared by shaving, an ECG was conducted to obtain heart rate and Q-wave, rats treated with endotracheal intubation via throat and a respiratory pump was connected to provide an artificial respiration with $100 \%$ oxygen, whose respiratory rate was 80 bpm and the tide volume was $0.7-0.8 \mathrm{~mL}$. The operative region was disinfected by iodine complex and the operation was started. The skin was incised at about $1.5 \mathrm{~cm}$ long on the left side between the third and fourth ribs transversely. After blunt separation of muscle layer, the chest was opened along the intercostal space. A thoracotomy device was used to expand the operative field. The pericardium was torn, then the left anterior descending artery can be exposed in the vision. The ligation sites lied in the distance from the junction of the arterial cone and left atrial appendage at $2 \mathrm{~cm}$, when the line 5/0 was used. Immediately after that, an ECG was done on the rat. If lead I showed that ST-segment elevated obviously ( $\geq 3 \mathrm{~mm})$, the chest wall was sutured layer by layer. The sham operation was the same as the method mentioned above except the step of ligation. All the rats were injected penicillin and given routine feeding as before after the operation. Twenty-four hours later, all the rats were put to death after having an ECG and the venous sampling via abdominal vein by which the troponin I (TnI) was tested.

\section{Statistical analysis}

Data were expressed as mean \pm SEM. $\mathrm{P}<0.05$ was considered significant. Results were compared using one-way ANOVA followed by Tukey's multiple comparison test.

\section{Results}

The ECG showed that all the rats in the AMI group had the

Table 2. The Blood Glucose Levels of the Different Groups

\begin{tabular}{lllllll} 
Groups & N & Before the operation & N & After the operation & N & Before the death \\
\hline MH & 11 & $17.71 \pm 1.99^{\mathrm{a}}$ & 10 & $18.87 \pm 9.37^{\mathrm{a}}$ & 11 & $6.36 \pm 0.74$ \\
$\mathrm{MN}$ & 13 & $7.68 \pm 4.93$ & 10 & $9.16 \pm 6.30$ & 10 & $6.19 \pm 0.45$ \\
ML & 15 & $3.13 \pm 0.41^{\mathrm{b}}$ & 15 & $4.50 \pm 5.31^{\mathrm{b}}$ & 2 & $6.40 \pm 0.14$ \\
$\mathrm{SH}$ & 12 & $17.66 \pm 2.56^{\mathrm{a}}$ & 11 & $21.20 \pm 8.75^{\mathrm{a}}$ & 11 & $6.15 \pm 0.76$ \\
$\mathrm{SN}$ & 12 & $6.48 \pm 0.94$ & 12 & $7.64 \pm 0.80$ & 9 & $6.66 \pm 0.50$ \\
SL & 16 & $3.41 \pm 0.28^{\mathrm{b}}$ & 13 & $6.09 \pm 3.95^{\mathrm{b}}$ & 17 & $6.30 \pm 0.71$ \\
Total & 79 & $8.60 \pm 6.46$ & 71 & $10.59 \pm 8.76$ & 60 & $6.32 \pm 0.65$ \\
\hline
\end{tabular}

aCompared with the normal and low blood glucose groups, the high blood glucose group had a higher blood glucose levels, which was statically significant $(\mathrm{P}<0.05)$. ${ }^{b}$ Compared with the normal and high blood glucose group, the low blood glucose groups had a lower blood glucose levels, which was statically significant $(P<0.05)$. MH: myocardial infarction high glucose level; MN: myocardial infarction normal glucose level; ML: myocardial infarction low glucose level; SH: sham-operated high glucose level; SN: sham-operated normal glucose level; SL: sham-operated low glucose level. 
Table 3. The Heart Rate of the Different Groups

\begin{tabular}{lllllll}
\hline Groups & N & Before the operation & N & After the operation & N & Before the death \\
\hline MH & 11 & $426.36 \pm 32.13$ & 11 & $443.55 \pm 43.77^{\mathrm{a}}$ & 10 & $484.70 \pm 23.59$ \\
MN & 12 & $433.50 \pm 31.00$ & 12 & $403.25 \pm 81.58$ & 12 & $426.67 \pm 125.25$ \\
ML & 15 & $427.00 \pm 42.00$ & 15 & $403.00 \pm 36.24$ & 15 & $453.73 \pm 35.79$ \\
SH & 11 & $383.18 \pm 40.81$ & 10 & $404.20 \pm 32.30$ & 12 & $425.33 \pm 123.43$ \\
SN & 12 & $417.42 \pm 43.34$ & 12 & $419.17 \pm 39.88$ & 11 & $466.64 \pm 41.52$ \\
SL & 17 & $435.47 \pm 37.51$ & 16 & $437.88 \pm 22.15^{\mathrm{b}}$ & 17 & $460.53 \pm 38.59$ \\
Total & 78 & $422.10 \pm 40.68$ & 76 & $418.96 \pm 47.09$ & 77 & $452.45 \pm 75.63$ \\
\hline
\end{tabular}

${ }^{a}$ Compared with the $\mathrm{MN}$ and $\mathrm{ML}$ groups, the $\mathrm{MH}$ group had a much faster heart rate, which was statically significant $(\mathrm{P}<0.05)$; also, the $\mathrm{MH}$ group had a much faster heart rate than before, which was statically significant $(\mathrm{P}<0.05)$. ${ }^{\mathrm{b}} \mathrm{Compared}$ with the $\mathrm{SL}$ group, the ML group had a lower heart rate, which was statically significant $(P<0.05)$. $\mathrm{MH}$ : myocardial infarction high glucose level; MN: myocardial infarction normal glucose level; ML: myocardial infarction low glucose level; SH: sham-operated high glucose level; SN: sham-operated normal glucose level; SL: sham-operated low glucose level.

common trait, the formation of Q-wave in more than three leads, while the sham group had none Q-wave in any lead or had the formation of Q-wave in less than two leads. Likewise, the results of the test of TnI lent credit to that the AMI group had much higher TnI than the sham group (Table 1). The difference was statistically significant $(\mathrm{P}<0.05)$. It was proved that the kinds of the groups were successfully prepared.

In sum, 43 rats died, of which 10 rats died from respiratory failure and five rats died from arrhythmia. It was found that 17 rats died in the hyperglycemic group. Other deaths occurred for no obvious reason.

The following were results about the levels of the blood glucose and the HR of the rats in the different groups.

The rats in the hyperglycemia group had a much higher blood glucose level than the other groups and the rats in the hypoglycemia group had a lower blood glucose level than the other groups; the difference was significant $(\mathrm{P}<0.05)$ (Table 2). It was proved that the kinds of the groups of different blood glucose levels were successfully prepared.

The HR of the rats in the hypoglycemic group is higher than that of the hyperglycemic group and normal blood glucose group before AMI, and the difference is significant $(\mathrm{P}<$ $0.05)$. The HR of the hyperglycemic rats is higher than that of the hypoglycemic and normal blood glucose rats after AMI, and the difference is significant $(\mathrm{P}<0.05)$. In the hypoglycemic group, the HR of the rats who suffered from AMI is lower than that of the rats of the sham group, and the difference is

Table 4. The Heart Rate Before the Operations

\begin{tabular}{lll}
\hline Groups & N & Blood glucose \\
\hline $\mathrm{H}$ & 22 & $404.77 \pm 42.105$ \\
$\mathrm{~N}$ & 24 & $425.46 \pm 37.753$ \\
$\mathrm{~L}$ & 32 & $431.50 \pm 39.235^{\mathrm{a}}$ \\
Total & 78 & $422.10 \pm 40.682$ \\
\hline
\end{tabular}

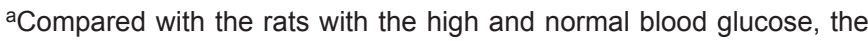
rats with low blood glucose had a more fast heart rate, which was statically significant $(\mathrm{P}<0.05)$. $\mathrm{H}$ : high glucose level; $\mathrm{N}$ : normal glucose level; L: low glucose level. significant $(\mathrm{P}<0.05)$ (Tables 3 and 4$)$.

\section{Discussion}

Over the years, since diabetes became an independent predictor among all the risk factors of coronary heart disease (CHD), abnormal blood sugar becomes the focus of experts [7, 8]. At the same time, it was verified that the increase of resting HR was related to the $\mathrm{CV}$ events based on a large number of Cohort researches and prospective double-blind clinical trials. So, more and more people begin to pay attention to the HR of the patients with AMI complicated with abnormal blood glucose [9-13].

At present, lots of studies involved the relationship between the HR turbulence (HRT) or HR variability (HRV) and blood sugar. HRT refers to sinus rhythm with a process from acceleration to deceleration after a single premature ventricular contraction (PVC), that is, it is the sensitive response of the sinoatrial node to the single premature ventricular contraction. In 1999, Jiang [14] proposed a new non-invasive examination index - HRT that was used to describe changes of HR of the PVC. What they thought that HRT can effectively predict the risk of the patients with myocardial infarction has been confirmed by a number of clinical studies. In the Chinese Heart Assembly, researches put forward that fasting blood glucose levels have association with HRT closely, and the patients with impaired glucose regulation already have cardiac autonomic dysfunction. HRV refers to the phenomenon that the sinus HR changes periodically in a certain period of time, which is an important index to reflect balance of the tension between sympathetic and parasympathetic. There are two methods to determine HRV - time domain and frequency domain. The clinical significance is that HRV, being in response to the activities of the autonomic nervous, is the indicator of vagal and sympathetic quantitatively. The reduction of HRV, the most valuable independent index to predict the malignant arrhythmia and sudden cardiac death, is close to severe arrhythmias and sudden cardiac death, which is more specific and sensitive than the late potential positive and the reduced ejection fraction. 
As a result, many scholars advocate the use of HPV to evaluate the function of the cardiac autonomic nerve quantitatively $[15,16]$. In their opinion, abnormal glucose level can induce HR disorder by cardiac sympathetic or parasympathetic dysfunction. $\mathrm{Wu}[17]$ analyzed the abnormal HRV in patients with fasting blood glucose and the influence of rosiglitazone on it, then they come to that the phenomenon of autonomic nerve damage existed in the patients with fasting blood glucose abnormalities, and the main performance is that the vagus nerve is abated, while the sympathetic is well, which disordered the balance between the sympathetic and vagal. Jamerson et al [18] pointed up in their experiment about the relationship between sympathetic overstimulation and insulin resistance that the excessive sympathetic excitation can lead to a faster HR, abnormal glucose metabolism and other disorders. Additionally, other scholar studies show that glucose fluctuations can damage the cardiac autonomic nervous function [19]. However, the exact mechanism remains to be confirmed by further clinical studies.

In our results based on animal study, the HR in the rats with hypoglycemia was generally fast; the HR in the rats with hyperglycemia and acute myocardial ischemia was faster. However, the HR of the rats with AMI was lower than those without myocardial infarction when they were in the same low glucose level. The possible mechanisms we considered were as follows: the response of the normal to the decrease of the blood glucose is that the secretion of insulin is reduced or completely suppressed, the secretion of hormone that increases blood sugar will increase, hypothalamus - adrenergic nerve excites and the cognitive disorder. Clinical manifestations may be divided into neurological symptoms and brain dysfunction symptoms. For the former, the neurologic symptoms often start quickly, which can be named neuroglycopenia. The reasons lie in that the central nervous system is most sensitive to hypoglycemia, but the nerve cells themselves have no glycogen reserves, in addition that the brain cells have to be dependent on the blood glucose completely to provide its energy. The suppressed performance of the impaired brain function generally occurs in sequence of cerebral cortex, subcortical central (including the basal ganglia), hypothalamus and autonomic nervous system, brain and other area. Among them, the hypothalamus may be considered as the central regulation of glucose metabolism. Many neurons in the hypothalamus contain sugar receptors, which can feel the change of the glucose concentration lied in the extracellular fluid. When the blood glucose lowers, the information produced by sugar sensing will convert to the relevant neurons quickly, which can excite corticotropin-releasing hormone $(\mathrm{CRH})$, thyrotropin-releasing hormone (TRH) and other cells in the hypothalamic, and promote the release of $\mathrm{CRH}$, TRH, and excitatory amino acid. Under the action of those substances mentioned above, pituitary-adrenal axis is activated to increase the secretion of glucocorticoids and adrenal medulla catecholamines. For another, hypoglycemia itself can stimulate the sympathetic nerve and the adrenal medulla cells release catecholamines, which leads to increasing HR. In 1951, Himwich [20] who divided the hypoglycemia into different periods according to the degree of the brain damage thought that when the cerebral cortex of telencephalon - diencephalon is damaged, there will have no sensory resolution, no reaction, fast $\mathrm{HR}$, and pupil dilation. While the neurons and life pivot are damaged, there will be coma, weak breathing, bradycardia, hypothermia, and so on. This gave a good explanation of the fasting HR at first of our experimental animals with hypoglycemia but slow at the onset of AMI. Some scholars think that hypoglycemia can cause released catecholamine substances, which aggravates the myocardial ischemia or may be the induced factor of arrhythmia. In addition, hypoglycemia can stimulate the secretion of growth hormone $(\mathrm{GH})$, cortisol, glucagon and epinephrine. That is to say, the normal rat has its own mechanism to regulate its blood sugar levels, which validates that a normal diet can help the rate suffer from acute hypoglycemia quickly, and along with the blood glucose response, the abnormal HR gradually returns to its original level. Some researchers have shown that the incidence of patients with AMI complicated with sinus tachycardia was $30 \%$. The main mechanism was that the excitability of the cardiac autonomic nerve caused by acute myocardial ischemia induced too fast heartbeat, which is also the important cause of CV events as well as sudden death.

When the rats with high blood glucose developed AMI, their HRs were increased obviously. There are two problems here. Firstly, there is some correlation between hyperglycemia and HR. Our statistical analysis of the data revealed weak positive correlation between them, but without statistical significance. Wei [9] found that with the increase of resting HR, fasting blood glucose levels are increased accordingly, which was also confirmed in many others studies [21-24]. Another researcher's experiment showed that the glucose level showed a linear or nearly linear upward trend with the increasing HR [25]. Secondly, HR and myocardial ischemia are related. The Gao's [26] experimental data showed that the HR in the rats with AMI was significantly higher than that in other groups, while Li [27] found in his study that the bias deviation of the value of ST segment, T-wave and HR was increased in the ischemic ST events, compared with the based value of the non-ischemic period of time. Therefore, we can come to the conclusion that hyperglycemia in rats with AMI can easily complicate with fast HR [28]. It is believed that the increasing HR, together with hyperglycemia and other metabolic abnormalities, was cause by dysfunction of autonomic nervous system and sympathetic activation, which was the pathophysiological basis to induce the CV events [29]. In Huram's study, he tried to illustrate to us that when the resting HR increased by $10 / \mathrm{min}$, a major CV event risk increased $8 \%$ in 9,580 patients with stable coronary heart disease with a mean follow-up of 4.9 years. When the resting HR was above $70 \mathrm{bpm}$, the risk of hospitalization caused by heart failure increased more than two times. HR is an objective indicator to reflect the tension of sympathetic and vagal. When sympathetic was activated, blood catecholamine concentration increased then, resulting in accelerated HR, whereas the over-sympathetic activity can lead to multiple metabolic abnormalities, including metabolic disorders, abdominal fat accumulation and insulin resistance, and the latter will make the heartbeat faster through sympathetic mediated. Therefore, the fast HR often complicates with hypertension, hyperglycemia, hyperlipidemia, obesity, high cholesterol and other risk factors. On the other hand, abnormal glucose metabolism stimulates insulin secretion and 
compensatory increase, causing hyperinsulinemia, which can also increase HR. In recent years, there is some correlationship among insulin resistance, glucose metabolism and lipid metabolism both in the domestic and foreign researches. The patients with AMI were in a state of stress, and plasma catecholamine increased sharply, which enhanced sympathetic nervous activity, and increased resting HR [30]. The levels of increased resting HR reflect the conditions of sympathetic activity. The higher the resting HR increased, the more excite of sympathetic nerve. However, the excessive sympathetic excitation can lead to the increasing myocardial oxygen consumption, which aggravates the myocardial hypoxic and lowers the threshold of arrhythmia, which can easily lead to heartbeat tachycardia [31]. HR is the important influence factor of myocardial oxygen consumption. When the HR increases, the myocardial oxygen supply reduces and the myocardial oxygen consumption increases, which in reverse aggravate the myocardial ischemia and necrosis. Early studies have confirmed that higher resting HR increases the risk of sudden death [32]. Some researchers found that by using $\beta$-blockers to reduce the myocardial oxygen consumption and sympathetic activity to control the HR of the patients with AMI to a reasonable level, the mortality was reduced accordingly $[33,34]$. In Marfella et al's study [35], they allowed the healthy volunteers to increase their blood sugar to acute hyperglycemia. Two hours later, he found the emergence of systolic and diastolic blood pressure, pulse, blood catecholamine concentration and QT interval prolongation. All the changes mentioned above can be corrected reversely along with the blood sugar return to be normal. Some scholars confirm that hyperglycemia can increase systolic and diastolic blood pressure, HR, and plasma catecholamine levels and decrease leg blood flow. And acute hyperglycemia may increase the production of free radicals that mediate its hemodynamic effects.

\section{Conclusion}

Hypoglycemia allows faster HR and the HR in the rats with hyperglycemia is higher than that in the rats with hypoglycemia among the AMI rats.

\section{Disclosures}

The author(s) declared no potential conflicts of interest with respect to the research, authorship, and/or publication of this article.

\section{Foundation}

The Beijing Heart and Metabolism Survey (BHMS) was supported by grants from the Beijing Nova Program (No. Z121107002512053), the Beijing Health System High Level Health Technology Talent Cultivation Plan (No. 2013-3013), Beijing Outstanding Talent Training Program (No. 2014000021223ZK32), the National Natural Science Founda- tion of China (No. 81100143), the Beijing Municipal Administration of Hospitals Clinical Medicine Development of Special Funding Support (No. ZYLX201303), and the National Key Clinical Speciality Construction Project.

\section{References}

1. Yang SW, Zhou YJ, Nie XM, Liu YY, Du J, Hu DY, Jia DA, et al. Effect of abnormal fasting plasma glucose level on all-cause mortality in older patients with acute myocardial infarction: results from the Beijing Elderly Acute Myocardial Infarction Study (BEAMIS). Mayo Clin Proc. 2011;86(2):94-104.

2. Yang SW, Zhou YJ, Hu DY, Nie XM, Liu YY, Hua Q, Wang $X$, et al. Association between admission hypoglycaemia and in-hospital and 3-year mortality in older patients with acute myocardial infarction. Heart. 2010;96(18):1444-1450.

3. Fox K, Borer JS, Camm AJ, Danchin N, Ferrari R, Lopez Sendon JL, Steg PG, et al. Resting heart rate in cardiovascular disease. J Am Coll Cardiol. 2007;50(9):823-830.

4. Graham I, Atar D, Borch-Johnsen K, Boysen G, Burell G, Cifkova R, Dallongeville J, et al. European guidelines on cardiovascular disease prevention in clinical practice: executive summary: Fourth Joint Task Force of the European Society of Cardiology and Other Societies on Cardiovascular Disease Prevention in Clinical Practice (Constituted by representatives of nine societies and by invited experts). Eur Heart J. 2007;28(19):2375-2414.

5. Liu Yuansheng, Chen Yunzhen. Experimental Model of Chronic Myocardial Infarction In Rats. Journal of Medical University of Chongqing. 2007;27(2):153-155.

6. WANG Yong, GAO Da-zhong, YIN Yue-hui, et al. Method selection for establishment of myocardial infarction model and the electrocardiographic presentation in the rats. Acta Laboratorium Animalis Scientia Sinca. $2011 ; 19(6)$.

7. Timmer JR, van der Horst IC, Ottervanger JP, Henriques JP, Hoorntje JC, de Boer MJ, Suryapranata H, et al. Prognostic value of admission glucose in non-diabetic patients with myocardial infarction. Am Heart J. 2004;148(3):399404.

8. SU Hui, SUN Xin, WANG Xiaoming, et al. Effects of hyperglycemia during myocardial ischemia on myocardial ischemia/reperfusion injury in rats. Chin J Mult Organ Dis Elderly. 2008;7(3):220-222.

9. Wei Xi. Difference of waist circumference, blood pressure, blood glucose and blood fat in healthy examinees with different resting heart rates and genders. Journal of Clinical Rehabilitative Tissue Engineering Research. 2007;2(12).

10. Benetos A. [Hypertension - heart rate and cardiovascular risk]. Arch Mal Coeur Vaiss. 2000;93(11 Suppl):1371-1376.

11. Zhang Luxing, Zheng Qiangsun, Li Bo, et al. Changes Of Resting Heart Rate In Patients With Coronary Heart Disease: First Level Evaluation Index Of Pathogenetic Condition And Prognosis. Chinese Journal of Clinical 
Rehabilitation. 2004;8(18):3504-3505.

12. Fox K, Ford I, Steg PG, Tendera M, Robertson M, Ferrari R. Relationship between ivabradine treatment and cardiovascular outcomes in patients with stable coronary artery disease and left ventricular systolic dysfunction with limiting angina: a subgroup analysis of the randomized, controlled BEAUTIFUL trial. Eur Heart J. 2009;30(19):2337-2345.

13. Heusch G. A BEAUTIFUL lesson - ivabradine protects from ischaemia, but not from heart failure: through heart rate reduction or more? Eur Heart J. 2009;30(19):23002301.

14. Jiang Xuejun. Progress In The Study Of Heart Rate Turbulence, 365 . Heart W001654.

15. Zhou Jian, Jia Weiping, Yu Ming, et al. Establishment of Normal Reference Value Of Glucose Dynamic Parameters The And Clinical Application. Chinese Journal of Department of Internal Medicine. 2007;46:189-192.

16. Zheng Yichen, Ni Liansong, Zhao Yuxiang, et al. Analysis on Heart Rate Variability in 65 Patients With Type 2 Diabetes. Chinese Journal of Practical Department of Internal Medicine. 2002;4(4):29.

17. Wu Weijun, Xiang Yanjun. Analysis on Heart Rate Variability In Patients With Abnormal Fasting Plasma Glucose And Its Influence Of Rosiglitazone. ZHEJIANG Journal of Clinical Medcine. 2007;10:1368-1369.

18. Jamerson KA, Nesbitt SD, Amerena JV, Grant E, Julius S. Angiotensin mediates forearm glucose uptake by hemodynamic rather than direct effects. Hypertension. 1996;27(4):854-858.

19. YANG Fei. Relecance analysis of heart rate cariability and blood-sugar cariance of the patients with type 2 diabetes mellitus. Chinese Journal of Cardiovascular Research. 2012;10(2):135-137.

20. Himwich HE. The somatic division of the central nervous system: studies through the symptoms of hypoglycemia and acute anoxia. In: Brain Metabolism and Cerebral Disorders. Baltimore: William \& Wilkins. 1951; p. 257.

21. Palatini P, Casiglia E, Julius S, Pessina AC. High heart rate: a risk factor for cardiovascular death in elderly men. Arch Intern Med. 1999;159(6):585-592.

22. Cooney MT, Vartiainen E, Laatikainen T, Juolevi A, Dudina A, Graham IM. Elevated resting heart rate is an independent risk factor for cardiovascular disease in healthy men and women. Am Heart J. 2010;159(4):612-619 e613.

23. Nauman J, Janszky I, Vatten LJ, Wisloff U. Temporal changes in resting heart rate and deaths from ischemic heart disease. JAMA. 2011;306(23):2579-2587.

24. Woodward M, Webster R, Murakami Y, Barzi F, Lam TH,
Fang X, Suh I, et al. The association between resting heart rate, cardiovascular disease and mortality: evidence from 112,680 men and women in 12 cohorts. Eur J Prev Cardiol. 2014;21(6):719-726.

25. CHENG Min, ZHANG Qian, CHENG Rong. Analysis of the relation between heart rate and cardiovascular and metabolic diseases. Modern Preventive Medicine. 2007;34(11):2051-2054.

26. Gao Shudan, Wu Yangjing. The relationship between st segment and $\mathrm{t}$ wave in the electrocardiogram of myocardial ischemia and the changes of heart rate. Guide of China Medicine. 2012;10(4):148-149.

27. LI Yan-jun, HONG Feng, YAN Hong, SONG Jin-zhong, LIU Qian-qian. Analysis on related changes among st segment, t wave and heart rate during ischemic st episodes. Space Medicine \& Medical Engineering. 2010;23(5):340344.

28. Jiao Yunli. The study of the correlation between stress hyperglycemia and acute myocardial infarction complicated with cardiac arrhythmia. China Modern Doctor. 2010;48(3):145-146.

29. Yan W, Zuo W, Lin Q. [Evaluation of autonomic nervous function with heart rate variability and cardiovascular reflex tests in type II diabetes mellitus patients]. Zhonghua Nei Ke Za Zhi. 2000;39(10):670-673.

30. Lu Wenxuan. The clinical significance of resting heart rate changes in patients with acute myocardial infarction. Internal Medicine of China. 2011;6(4):314-315.

31. Zhu Qiaoping, Li Jiansheng, Wang Chunlian, et al. The Relationship Between Sympathetic Activity And Ventricular Arrhythmia In Patients With Acute Myocardial Infarction. Clinical Medicine of China. 2003;19(2):117118.

32. Duan Lin. Correlation Analysis Between Heart Rate Variability And Resting Heart Rate In Elderly Patients With Coronary Heart Disease. The Journal of Medical Theory and Practice. 2010;23(3):288-289.

33. Zhu Qiaoping, Huang Jianhua, Ruan Jianghua. Effects Of Metoprolol On Autonomic Nerve In Patients With Acute Myocardial Infarction. Journal of Practical Electrocardiology. 2005;14(1):21-22.

34. Liu Jin, Zhou Lin, Liu Keying. Relations Between Different Heart Rate And Prognosis In Patients With Acute Myocardial Infarction. The Journal of Practical Medicine. 2009;25(17):2880-2883.

35. Marfella R, Nappo F, De Angelis L, Paolisso G, Tagliamonte MR, Giugliano D. Hemodynamic effects of acute hyperglycemia in type 2 diabetic patients. Diabetes Care. 2000;23(5):658-663. 